\title{
Recent Results on Soft QCD at ATLAS
}

\author{
David Milstead (on behalf of the ATLAS Collaboration) $)^{\mathrm{a}, *}$ \\ ${ }^{a}$ Fysikum, Stockholms Universitet, Stockholm, Sweden.
}

\begin{abstract}
The ATLAS experiment at the Large Hadron Collider has produced a number of measurements of final state observables which are sensitive to soft processes mediated by the strong force. In this article, measurements of identified particle production $(\phi(1020))$ are presented as are studies of the underlying event and double parton scattering.
\end{abstract}

Keywords:

\section{Introduction}

An important component of the physics program of the ATLAS experiment [1, 2] at the Large Hadron Collider is the study of the strong force. Although the theory of perturbative QCD has survived a number of precision tests, these correspond to short distance $(<<\mathrm{fm})$ processes. Interactions taking place over longer distance scales can not be reliably predicted. Progress in this area has traditionally depended on a symbiosis of precision measurement and phenomenology. In these proceedings, a number of measurements which are sensitive to soft processes are presented.

\section{Measurement of $\phi(1020)$ production}

The decay mode $\phi \rightarrow K^{+}+K^{-}$was used to reconstruct and measure the production of $\phi(1020)$ mesons from $p p$ collisions at a centre-of-mass energy of $7 \mathrm{TeV}$ in a minimum bias dataset corresponding to an integrated luminosity of $383 \mu \mathrm{b}^{-1}$ [3]. The kaons were identified using measurements of specific ionisation energy loss $\frac{d E}{d x}$. Measurements of average $\frac{d E}{d x}$ as a function of the charge-signed momentum of reconstructed charged particles are shown in Figure 1. Clearly separated bands due to different types of light hadrons are visible. The requirement of a highly pure sample of kaons restricts the kinematic range of the kaons and $\phi$-mesons which can be selected. Consequently, the results on $\phi$ production discussed in this section correspond to the following kinematic domain: $500 \mathrm{MeV}<p_{T, \phi}<1200 \mathrm{MeV}$,

\footnotetext{
* Speaker

Email address: milstead@physto. se (David Milstead (on behalf of the ATLAS Collaboration))
}

Preprint submitted to Nuc. Phys. (Proc. Suppl.) $\left|y_{\phi}\right|<0.8, p_{T, K}>230 \mathrm{MeV}$ and $p_{K}<800 \mathrm{MeV}$, where $p_{T, \phi}, y_{\phi}, p_{T, K}$, and $p_{K}$ are, respectively, the transverse momentum and rapidity of the $\phi$, and the transverse momentum and momentum of the kaons.

Fits were made to dikaon spectra to estimate the $\phi$ yield. A sample dikaon spectrum is shown in Figure 2. A normal distribution with a freely floating mean and standard deviation was used for the signal. The following empirically based function was used to parameterise the background:

$$
\begin{aligned}
& f_{\mathrm{BKG}}(m)=\left(1-e^{\left(2 m_{K}-m\right) / C}\right) \cdot\left(\frac{m}{2 m_{K}}\right)^{A} \\
& +B\left(\frac{m}{2 m_{K}}-1\right)
\end{aligned}
$$

Here, $m_{K}$ is the charged kaon mass, $m$ is the dikaon mass, and $A, B$, and $C$ are constants governing the background shape. Differential cross sections for $\phi$ production as a function of transverse momentum and rapidity are shown in Figures 3 and 4, respectively. The predictions of various QCD-based models are also shown. The best descriptions are provided by the PYTHIA 6 [4] tune DW and the Epos-LHC tune [5, 6]. The Pythia 6 MC09 tune prescribes slightly greater yields than are observed the data. The Pythia 6 Perugia0 tune predictions underestimate the data by a factor of two. Two Pythia 8 [7] A2 tunes, made with different parton distribution functions, also underestimate the data. The Herwig $++[8]$ model describes well the cross section for $p_{T \phi}<700 \mathrm{MeV}$ and $\left|y_{\phi}\right|>0.6$ but predicts a transverse momentum spectrum which falls too quickly.

September 5, 2014 


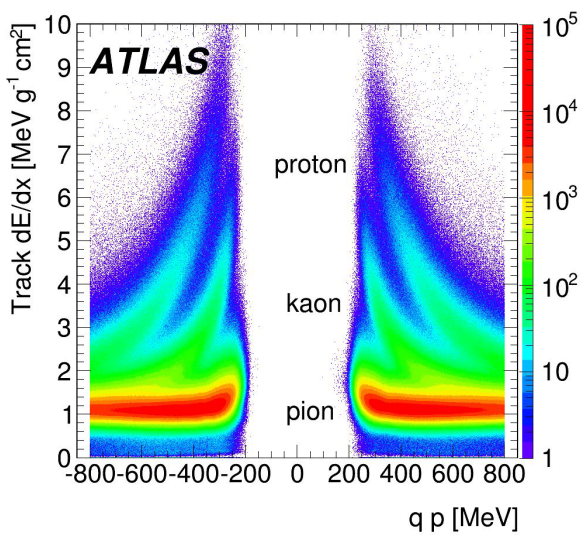

Figure 1: Measurements of the specific ionisation energy loss of tracks as a function of $q p$ where $q$ and $p$ are the reconstructed charge and momentum of the track. Bands are associated with various types of light hadrons. This figure is from Ref. [3].

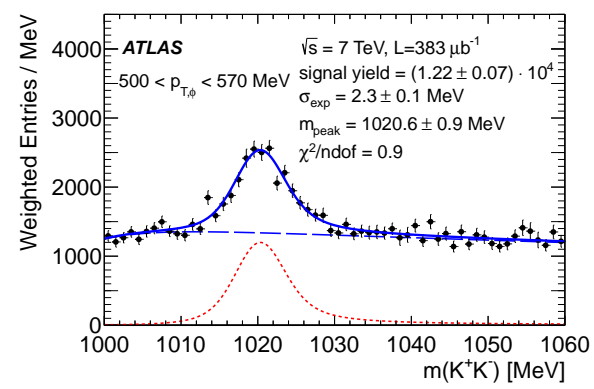

Figure 2: An example of the dikaon invariant mass spectrum in the data compared to results of the fit for a sample $p_{T \phi}$ bin. The dashed curve shows the background contribution and the dotted curve demonstrates the signal contributions. This figure is from Ref. [3].

A direct comparison with results on $\phi$ production from ALICE is made possible via an extrapolation of the ATLAS results to the fiducial region used by ALICE: $500<p_{T, \phi}<1200 \mathrm{MeV}$ and $\left|y_{\phi}\right|<0.5$. QCDbased models are used to perform this extrapolation. An additional systematic uncertainty of $\sim 10 \%$ due to different extrapolation predictions is assigned to the data. The ATLAS and ALICE differential cross sections as a function of $p_{T, \phi}$ are shown in Figure 5. The data from the two experiments agree well.

\section{Underlying event studies}

Soft and hard processes contribute to the so-called underlying event (UE). Experimental studies of the UE possess discriminating power between the various QCD-based models which seek to describe final states at the LHC. In addition to being an important research

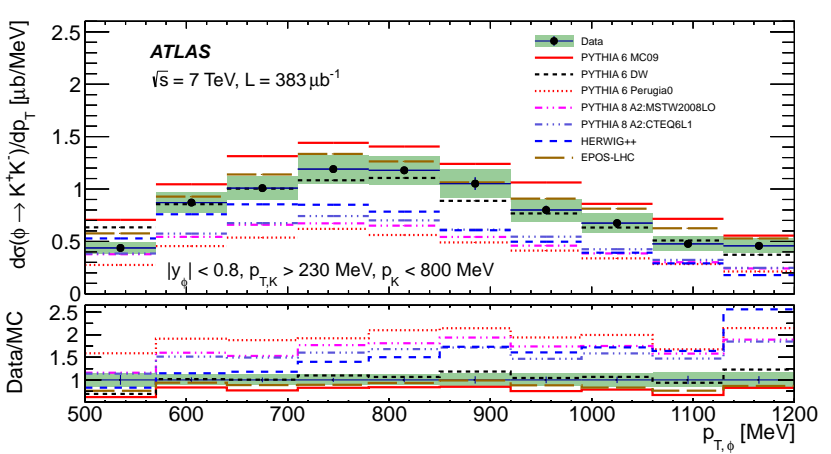

Figure 3: The $\phi \rightarrow K^{+} K^{-}$cross section in the fiducial region, with $500<p_{T, \phi}<1200 \mathrm{MeV},\left|y_{\phi}\right|<0.8, p_{T, K}>230 \mathrm{MeV}$ and kaon momentum $p_{K}<800 \mathrm{MeV}$, as a function of $p_{T, \phi}$. This figure is from Ref. [3].

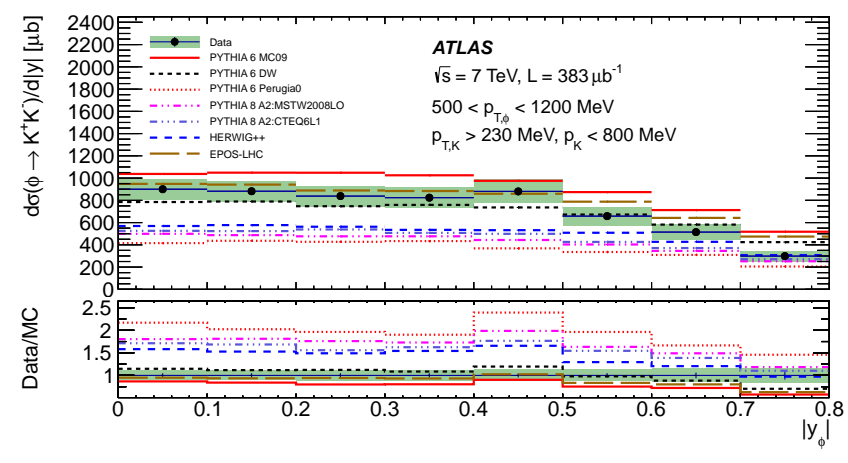

Figure 4: The $\phi \rightarrow K^{+} K^{-}$cross section in the fiducial region, with $500<p_{T, \phi}<1200 \mathrm{MeV},\left|y_{\phi}\right|<0.8, p_{T, K}>230 \mathrm{MeV}$ and kaon momentum $p_{K}<800 \mathrm{MeV}$, as a function of rapidity. This figure is from Ref. [3].

topic in its own right, a good understanding of the UE is necessary when searching for or measuring processes at short distance scales. A recent work from ATLAS [9], based on $p p$ collisions at a centre-of-mass energy of $7 \mathrm{TeV}$ and corresponding to an integrated luminosity of $37 \mathrm{pb}^{-1}$, measured the UE in jet events.

Regions which are azimuthally transverse to the hardest jet in an event are expected to be most sensitive to UE effects. In this study the leading jet possesses a value of transverse momentum between 20 and $800 \mathrm{GeV}$. The azimuthal regions are defined with respect to the $\phi$ angle of the leading jet, as sketched in Figure 6. Differential distributions of final state observables are made with respect to the different regions, including the two transverse regions. In the following, the so-called trans-max (trans-min) region corresponds to that transverse region in which an observable shows the largest (smallest) activity on an event-by-event basis.

The charged particle mean $p_{T}$ and densities of all- 


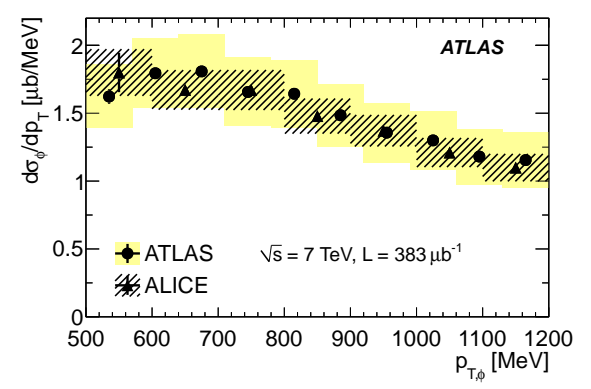

Figure 5: The $\phi \rightarrow K^{+} K^{-}$differential cross section as a function of $p_{T, \phi}$ is shown in the ALICE fiducial region. Extrapolated ATLAS results and ALICE data are shown. This figure is from Ref. [3].

particle transverse energy $E_{T}$ were measured in these regions, as were the charged particle multiplicity and particle $p_{T}$.

In Figure 7 distributions are shown of charged particle multiplicity $\frac{1}{N} \frac{d N}{\frac{N_{c h}}{\delta \eta \delta \phi}}$ and the scalar transverse momentum sum $\frac{1}{N} \frac{d N}{\frac{2 p T}{\delta \eta \delta \phi}}$ in the transverse, trans-max and transmin regions for different values of the leading jet transverse momentum $p_{T}^{\text {lead }}$. The data show growing hard tails in the spectra as $p_{T}^{\text {lead }}$ increases.

Further distributions, are shown in Figure 8. Here $\frac{\Sigma p_{T}}{\delta \eta \delta \phi}$ and $\frac{N_{c h}}{\delta \eta \delta \phi}$ are shown for inclusive jet events and exclusive dijet events. For the inclusive jet events, the trans-max regions shows growth with greater activity with leading jet momentum, whereas the trans-min region is almost constant. This result is consistent with the trans-min region being less affected by the hard component of the UE. A comparison with Pyтhia 6 using the Perugia tune is given. This model reproduces the main features of the distribution.

\section{Measurement of double parton interactions}

The prospect of double parton interactions (DPI) in scattering processes involving hadrons has long been considered theoreticaly [10-12]. Using a dataset taken in 2010 at a $7 \mathrm{TeV}$ centre-of-mass energy and with an integrated luminosity of $36 \mathrm{pb}^{-1}$, the ATLAS experiment has estimated the fraction of DPI events for $W+2$-jet processes for which muonic and electronic $W$-decays are sought [13]. This fraction can be used to estimate the value of $\sigma_{e f f}$, an effective area parameter for DPI.

The quantity $\Delta_{\text {jets }}^{n}$ is a sensitive observable from which the DPI fraction can be obtained. This quantity is defined as $\Delta_{\text {jets }}^{n}=\frac{\left|p_{T}^{J 1}+p_{T}^{J 2}\right|}{\left|p_{T}^{J 1}\right|+\left|p_{T}^{J^{2}}\right|}$, where $p_{T}^{J 1}$ and $p_{T}^{J 2}$ are the transverse momenta of the two leading jets in the event

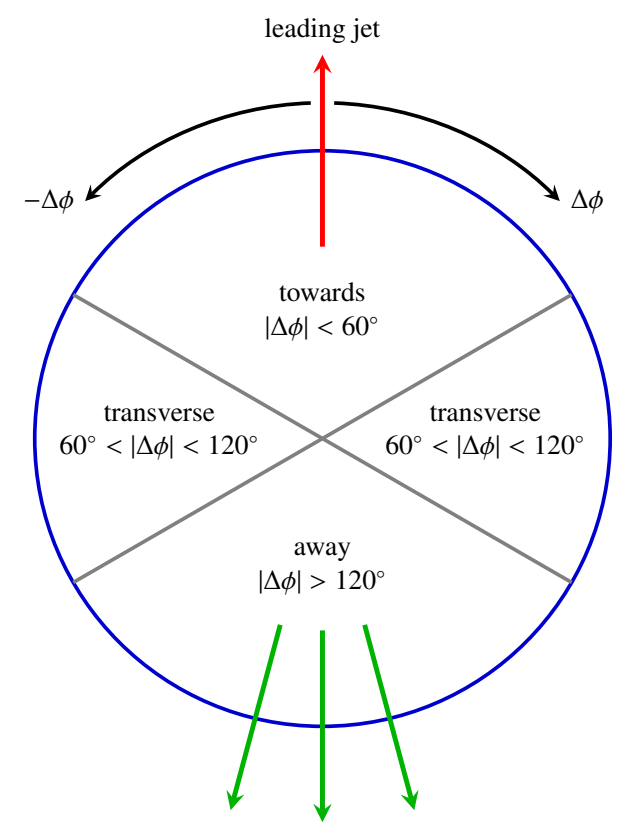

Figure 6: Illustration of regions in the azimuthal angle with respect to the leading jet. This figure is from Ref. [9].

and $\left|p_{T}^{J 1}+p_{T}^{J 2}\right|$ is the absolute value of the vector sum of the transverse components of the two jets.

Figure 9 shows two distributions of $\Delta_{\text {jets }}^{n}$ using a combined set of models: Alpgen [14], Herwig [15] AND JIMMY [16]. In one distribution, DPI may take place and for the other DPI is explicitly turned off. The ratio of the two distributions is also shown. The effect of DPI is most visible at low values of $\Delta_{j e t s}^{n}$. This is typically due to gluon-gluon fusion processes in DPI which would lead to a lower value of $\frac{\left|p_{T}^{J 1}+p_{T}^{J 2}\right|}{\left|p_{T}^{J 1}\right|+\left|p_{T}^{J 2}\right|}$ as the jets are produced more back-to-back than in the single parton scattering process.

Following optimisation of relevant DPI parameters, the prediction of $\mathrm{A}+\mathrm{H}+\mathrm{J}$ with DPI off was used a template for single parton interactions (template A). Template B, corresponding to DPI-only was taken from dijet data. The fraction of DPI was found to be $f_{D P}^{D}=$ $0.08 \pm 0.01 \pm 0.02$, where the two quoted uncertainties are due to statistical and systematic effects, respectively. The distribution of $\Delta_{\text {jets }}^{n}$ in data is shown in Figure 10 together with the fitted contributions of single particle interactions and DPI. Also shown is the prediction of $\mathrm{A}+\mathrm{H}+\mathrm{J}$ with DPI on.

A value of $\sigma_{e f f}$ of $15 \pm 3_{-3}^{+5} \mathrm{mb}$ can then be inferred. The evolution of $\sigma_{e f f}$ with centre-of-mass energy for different collision environments and processes is given in Figure 11. The ATLAS value agrees well with earlier 


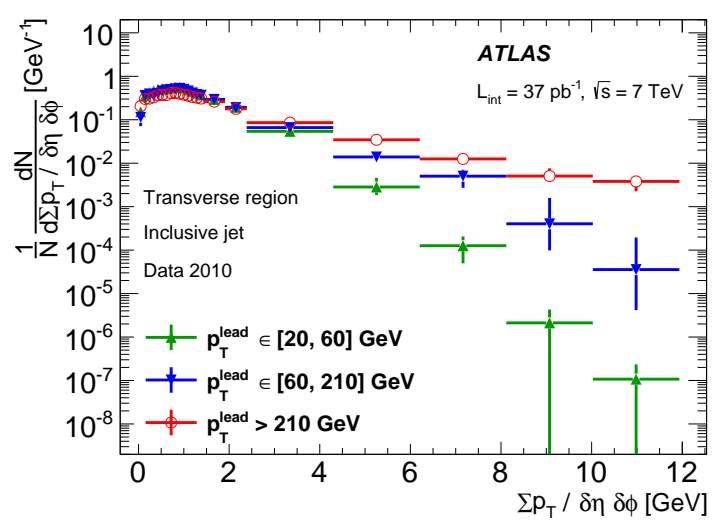

(a)

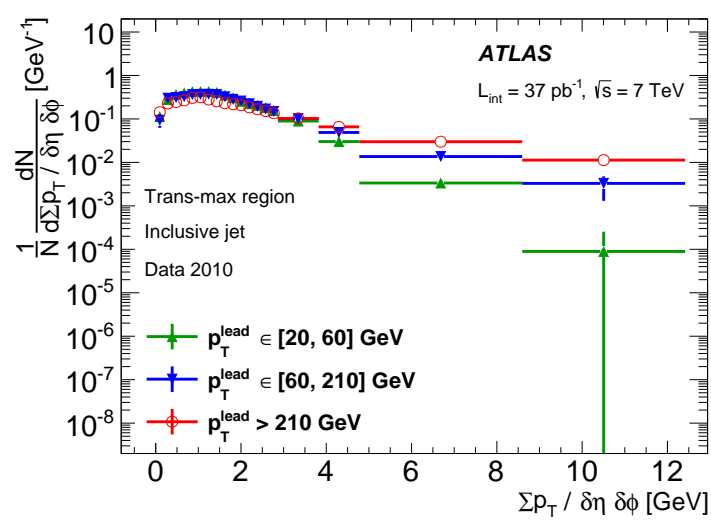

(c)

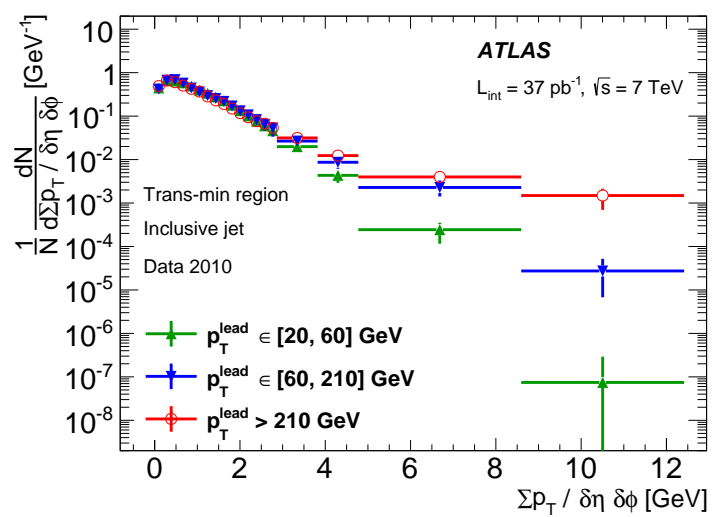

(e)

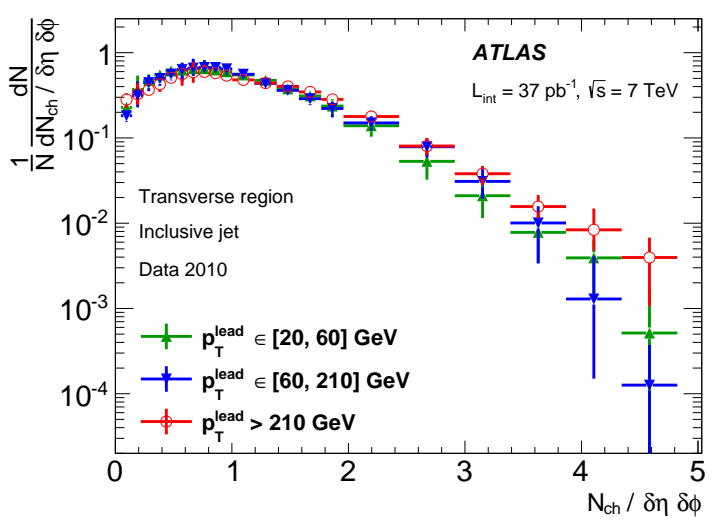

(b)

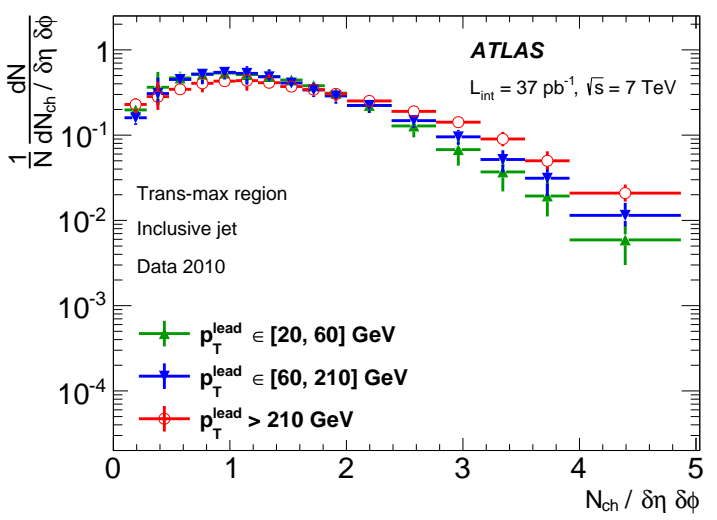

(d)

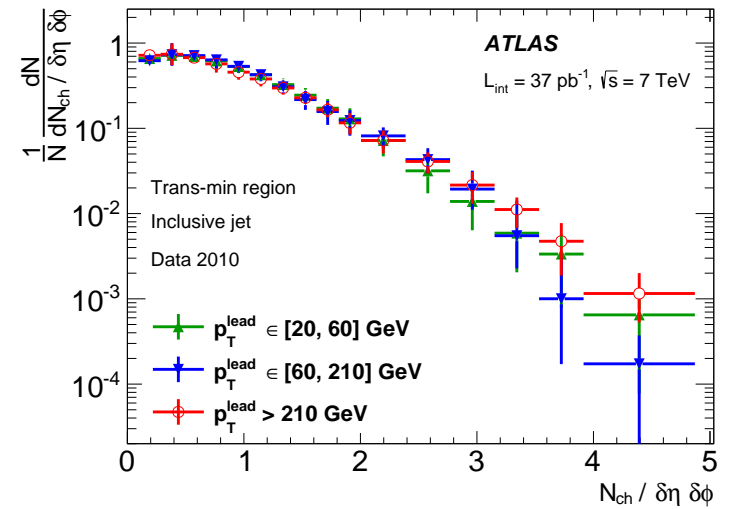

(f)

Figure 7: Comparison of transverse momentum sum (left) and charged particle multiplicity (right) density spectra for various selections on leading jet transverse momentum. This figure is from Ref. [9]. 


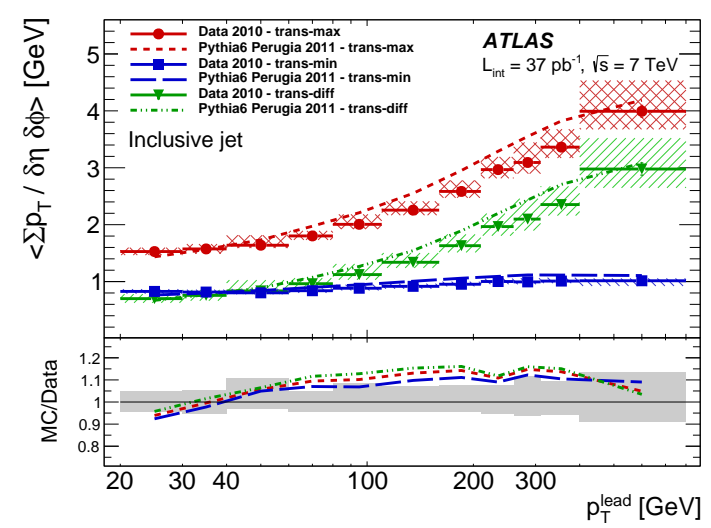

(a)

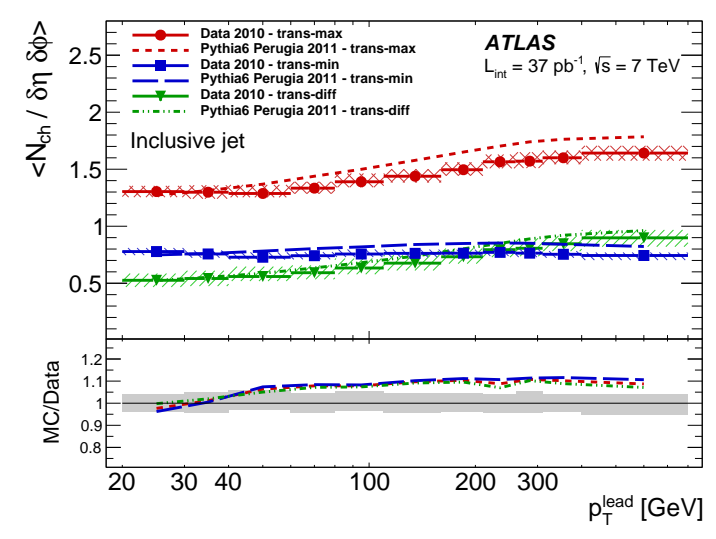

(c)

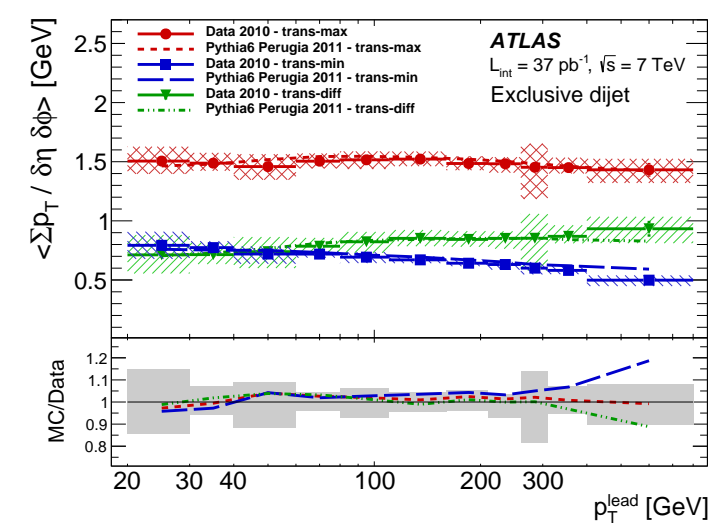

(b)

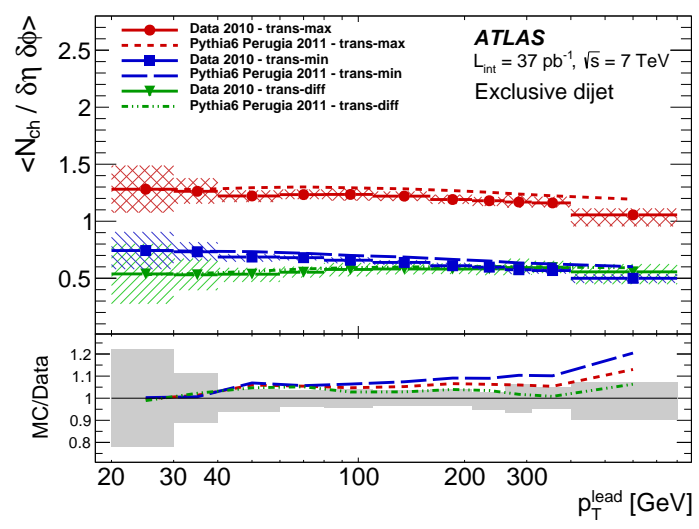

(d)

Figure 8: Profiles of charged particle $\Sigma p_{T}$ and multiplicity as a function of the leading jet $p_{T}$ for an inclusive jet selection (left) and exclusive dijet selection (right). This figure is from Ref. [9]. 


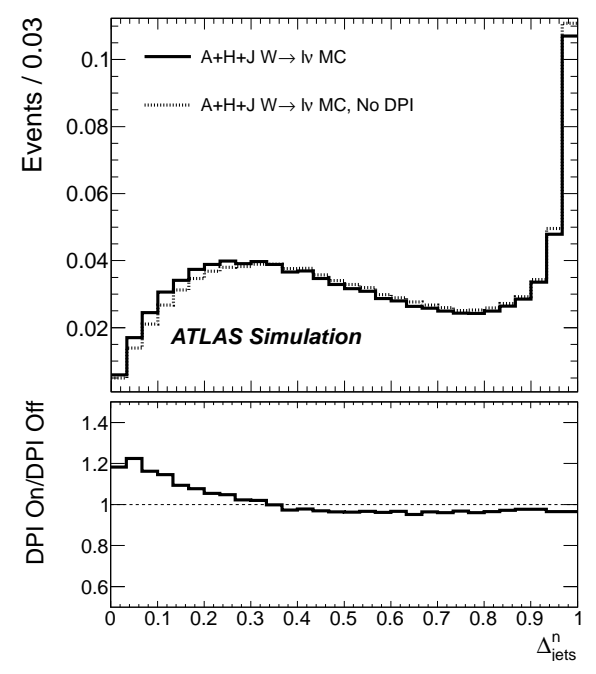

Figure 9: Top: predictions of the $\Delta_{\text {jets }}^{n}$ spectra with and without DPI turned on in the simulation. Bottom: the ratio of the two distributions shown above. This figure is from Ref. [13].

measurements at the $\mathrm{TeV}$ scale.

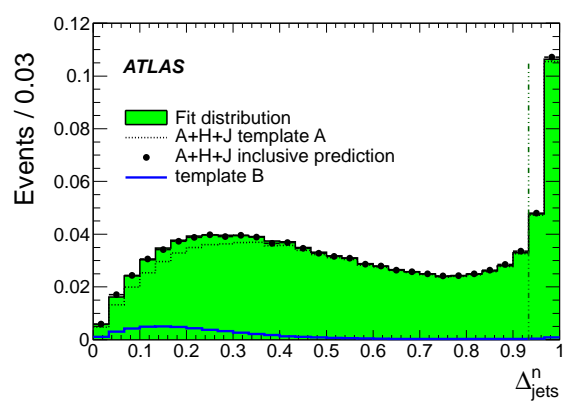

Figure 10: Distribution of $\Delta_{\text {jets }}^{n}$ in data. Also shown are the two templates used in the estimation of the fraction of DPI and the prediction from the $\mathrm{A}+\mathrm{H}+\mathrm{J}$ set of models. This figure is from Ref. [13].

\section{Conclusions}

Measurements from the ATLAS experiment at the LHC which are sensitive to soft processes have been presented.

\section{Acknowledgements}

The author would like to thank the organisers for a hospitable, stimulating and well run conference.

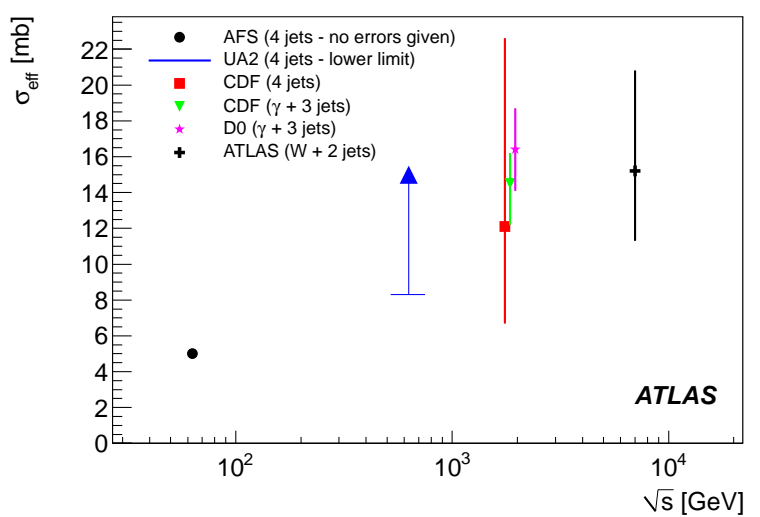

Figure 11: Values of $\sigma_{e f f}$ as extracted from different processes at various centre-of-mass energies. This figure is from Ref. [13].

\section{References}

[1] ATLAS Collaboration, JINST 3 (2008) S08003.

[2] ATLAS Collaboration, arXiv:0901.0512 [hep-ex].

[3] ATLAS Collaboration, Eur. Phys. J. C 74 (2014) 2895 [arXiv:1402.6162 [hep-ex]]. [4]

[4] T. Sjostrand, S. Mrenna and P. Z. Skands, JHEP 0605 (2006) 026 [hep-ph/0603175].

[5] K. Werner, I. Karpenko, T. Pierog, M. Bleicher and K. Mikhailov, Phys. Rev. C 82 (2010) 044904 [arXiv:1004.0805 [nucl-th]].

[6] K. Werner, F. M. Liu and T. Pierog, Phys. Rev. C 74 (2006) 044902 [hep-ph/0506232].

[7] T. Sjostrand, S. Mrenna and P. Z. Skands, Comput. Phys. Commun. 178 (2008) 852 [arXiv:0710.3820 [hep-ph]].

[8] M. Bahr, S. Gieseke, M. A. Gigg, D. Grellscheid, K. Hamilton, O. Latunde-Dada, S. Platzer and P. Richardson et al., Eur. Phys. J. C 58 (2008) 639 [arXiv:0803.0883 [hep-ph]].

[9] ATLAS Collaboration, Eur. Phys. J. C 74 (2014) 2965 [arXiv:1406.0392 [hep-ex]].

[10] P. V. Landshoff and J. C. Polkinghorne, Phys. Rev. D 18 (1978) 3344.

[11] F. Takagi, Phys. Rev. Lett. 43 (1979) 1296.

[12] C. Goebel, F. Halzen and D. M. Scott, Phys. Rev. D 22 (1980) 2789.

[13] ATLAS Collaboration, New J. Phys. 15 (2013) 033038 [arXiv:1301.6872 [hep-ex]].

[14] M. L. Mangano, M. Moretti, F. Piccinini, R. Pittau and A. D. Polosa, JHEP 0307 (2003) 001 [hep-ph/0206293].

[15] G. Corcella, I. G. Knowles, G. Marchesini, S. Moretti, K. Odagiri, P. Richardson, M. H. Seymour and B. R. Webber, JHEP 0101 (2001) 010 [hep-ph/0011363].

[16] J. M. Butterworth, J. R. Forshaw and M. H. Seymour, Z. Phys. C 72 (1996) 637 [hep-ph/9601371]. 